METAANALYSE

\section{Psoriasispatienten schweben in Diabetesgefahr}

Prävalenz- und Inzidenzraten von Diabetes liegen bei Patienten mit Psoriasis deutlich höher als bei Personen, die nicht an der Hautkrankheit leiden, haben Forscher der University of California, Campus Davis in einer Metaanalyse von 27 Beobachtungsstudien zur Diabetesprävalenz und -inzidenz bei Psoriatikern herausgefunden. Die Prä- valenz war um 59\% erhöht, wobei die Steigerung bei milder Psoriasis $53 \%$ und bei schweren Verlaufsformen $97 \%$ betrug. Die Inzidenz lag um $27 \%$ höher, wovon allerdings nur Patienten unter 60 Jahren betroffen waren.

Arch Dermatol 2012; doi:10.1001/2013.

jamadermatol.406

\section{TUMORTHERAPIE}

\section{Schweres Geschütz}

Am Heidelberger lonenstrahl-Therapiezentrum (HIT) wurde jetzt eine rotierende Schwerionenquelle (Gantry) in Betrieb genommen. Sie kann Tumorgewebe von Krebskranken aus allen Winkeln mit messerscharfer Genauigkeit treffen und eröffnet neue Therapieoptionen wie die Bestrahlung bei ungünstig lokalisierten Tumoren im Gehirn, in der Wirbelsäule, im Pankreas oder in der Lunge. Gantry ist das weltweit größte Gerät dieser Art.

Ärzte Zeitung vom 29.10.2012

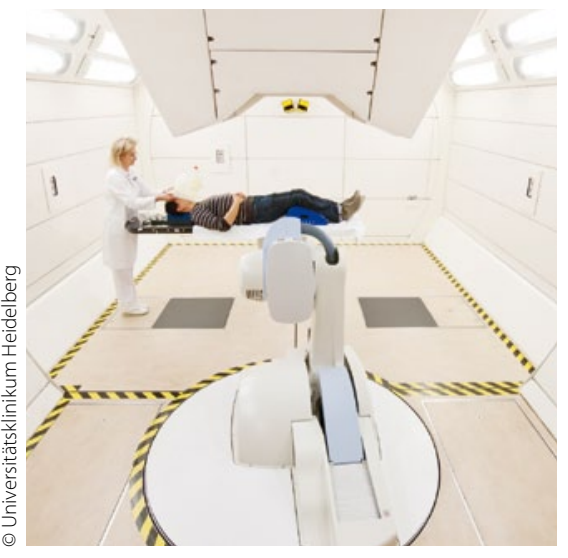

Der Patient trägt während der Bestrahlung eine Kunststoffmaske.

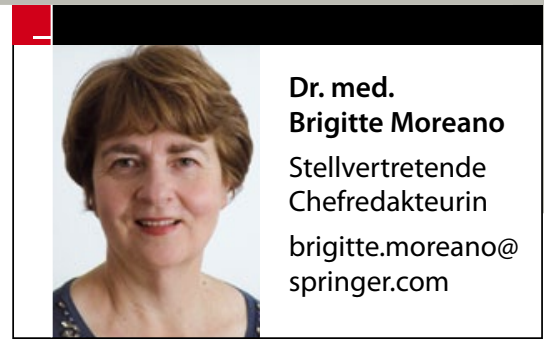

KOMBINIERTE RISIKEN

\section{Viererbande macht die Beinarterien dicht}

Rauchen, Hypertonie, Hypercholesterinämie und Diabetes gelten als Hauptverursachern peripherer Arterienverschlüsse. $\mathrm{Zu}$ Recht, wie eine US-Studie nun untermauert hat. Forscher der Harvard Medical School in Boston hatten 1986 mehr als 50000 Männer im Alter zwischen 40 und 75 Jahren in der "Health Professionals Follow-up Study" erfasst. Alle zwei Jahre wurden die medizinischen Daten der Probanden aktualisiert. Insgesamt wurden in der Studienkohorte 537 PAVK-Fälle aufgenommen. Bei 96\% aller PAVK-Diagnosen war mindestens einer der vier Risikofaktoren im Spiel. Statistisch gesehen ließe sich die PAVK-Inzidenz um $75 \%$ senken, würden die Einflüsse von Rauchen, Hypertonie, Hypercholesterinämie und Diabetes eliminiert.

JAMA 2012; 308: 1660-1667

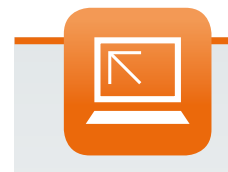

Neu auf springermedizin.de

Warum sich sexuell erregte Frauen weniger ekeln, Männern beim Pornogucken der Kopf schwellen kann, was Sexting ist und welche Folgen es für Jugendlichen hat Antworten auf diese und viele weitere Fragen finden Sie im neuen Schwerpunkt „Sexualität auf www.springermedizin.de/ sexualitaet 